\title{
BMJ Open Sensor technology to monitor health, well-being and movement among healthcare personnel at workplace: a systematic scoping review protocol
}

\author{
Maritta Välimäki (D) , , ${ }^{1,3}$ Kirsi Hipp (D) ,2 Jiarui Chen, ${ }^{1,3}$ Xiaoting Huang, ${ }^{1,3}$ \\ Jia Guo (D) , ${ }^{1,3}$ Man Sing Wong ${ }^{4}$
}

To cite: Välimäki M, Hipp K, Chen J, et al. Sensor technology to monitor health, well-being and movement among healthcare personnel at workplace: a systematic scoping review protocol. BMJ Open 2021;11:e054408. doi:10.1136/ bmjopen-2021-054408

- Prepublication history and additional supplemental material for this paper are available online. To view these files, please visit the journal online (http://dx.doi.org/10.1136/ bmjopen-2021-054408)

Received 21 June 2021 Accepted 08 October 2021

Check for updates

(C) Author(s) (or their employer(s)) 2021. Re-use permitted under CC BY-NC. No commercial re-use. See rights and permissions. Published by BMJ.

${ }^{1}$ Xiangya School of Nursing, Central South University, Changsha, Hunan, China ${ }^{2}$ Department of Nursing Science, University of Turku, Turku,

Finland

${ }^{3}$ Xiangya Centre for Evidence-

Based Nursing Practice \&

Healthcare Innovation, A JBI Affiliated Group, Changsha,

China

${ }^{4}$ Department of Land Surveying and Geo-Informatics, The Hong Kong Polytechnic University, Hong Kong, China

Correspondence to Professor Maritta Välimäki; maritta.vaelimaeki@csu.edu.cn

\section{ABSTRACT}

Introduction The well-being and health of healthcare personnel is becoming increasingly important in the delivery of high-quality healthcare. The recent developments in technology have provided new opportunities for the objective detection of a wide variety of real-world properties and movement. However, technologies that are used to monitor health, well-being and movement among healthcare personnel have not been fully synthesised. The overall aim of this scoping review is to examine what type of sensor technology is available to monitor the health, well-being and movement of healthcare personnel in healthcare settings. More specifically, we want to explore what types of sensor technology applications, for what purposes and how they have been used to monitor health, well-being and movement among healthcare personnel in different workplace settings.

Methods and analysis This scoping review protocol will follow Arksey and 0'Malley's methodology, complemented by the approach of the Joanna Briggs Institute to scoping reviews and guidance for conducting systematic scoping reviews. Peer-reviewed literature will be identified using a search strategy developed by a librarian, and a wide range of electronic datasets of medical, computer and information systems disciplines will be used. Eligibility of the articles will be determined using a two-stage screening process consisting of (1) a title and abstract scan, and (2) a full-text review. Extracted data will be thematically analysed and validated by an expert of sensor technology and a group of nurses as stakeholders. Descriptive statistics will be calculated when necessary. Ethics and dissemination The results obtained from the review will inform what technology has been used, how it has been used in healthcare settings and what types of technology might still be needed for future innovations. Findings of the scoping review will be published in a peerreviewed journal.

Registration This review was submitted in 0pen Science Framework on 12 December 2020.

\section{INTRODUCTION}

Over 59 million healthcare personnel work in various healthcare settings all over the world. ${ }^{1}$ Current improvements in the development
Strengths and limitations of this study

- This scoping review will contribute to strengthen the evidence base on sensor technology available to monitor healthcare personnel's health, well-being and movement in workplace settings.

- A comprehensive search for earlier published reviews and registered review protocols was conducted to justify this review and reduce the possibility of duplication.

- We will synthesise the evidence from published empirical studies with different designs, but publication bias can be caused by not considering grey literature for inclusion.

- A stakeholder group including nurse leaders has been formed for this scoping review and their opinions will be consulted in the different phases of the review process.

- Preferred Reporting Items for Systematic Reviews and Meta-Analyses Extension for Scoping Reviews checklist will be used to ensure detailed reporting.

of healthcare systems and technology have increased the costs and complexity of health services. ${ }^{2}{ }^{3}$ Changes in the ecosystem of healthcare have raised expectations of competence in personnel to manage complex work environments. ${ }^{45}$ At the same time, the wellbeing of healthcare staff continues to be at risk due to changes in work environments, ${ }^{6}$ which have contributed negatively to staff's physical and mental health. ${ }^{7-9}$ Good evidence already exists that the well-being of healthcare personnel should be on the agendas of healthcare organisations as well-being among staff is an important contributor to the quality of care. ${ }^{710-12}$

Well-documented literature has shown a variety of risk factors associated with health and well-being among healthcare personnel, such as heavy workloads, ${ }^{11} 13 \quad 14$ organisational problems ${ }^{11} 1^{13} \quad 15 \quad 16$ and leadership styles. ${ }^{217-20}$ Problems in social environments ${ }^{11}$ 
and workplace violence and harassment ${ }^{21} 22$ have also been identified as risks for the well-being and health of personnel. These problems have been documented as resulting in physical illnesses, psychological symptoms, ${ }^{23}$ burnout, ${ }^{8}$ low work satisfaction ${ }^{7}$ and quality of life, and increased sickness absence ${ }^{1119}$ among personnel. Despite the strong measurement trends, awareness of personnel health and well-being in the workplace is still not ubiquitous.

Various sets of instruments for self-reported measures have already been used to measure health and wellbeing. ${ }^{24}$ The most common methods are subjective survey measures. ${ }^{12}$ Although some of these measures may be considered outdated based on current standards, a few large-scale epidemiological cohort studies have captured detailed and long-term information on psychological and social factors in conjunction with rigorous assessment of healthcare personnel behaviour and health. ${ }^{14}$ Still, a variety of limitations of self-measures and survey measures have been identified. Subjective measures lay on individuals' interpretations, ${ }^{25}$ which can be affected by multiple contextual factors. ${ }^{22}$ Assessment results can also be vulnerable due to memory biases. ${ }^{1222} 26$ Cross-sectional survey instruments can only provide data depending on the timing of the data collection. ${ }^{22}$ In addition, low response rates may cause limitations in results. ${ }^{27}$ Because of these limitations, more usable and updated methods for assessing health and well-being among healthcare personnel are needed.

The use of sensor technology is growing as it provides new opportunities for more objective, accurate, updated and ongoing measurements of real-life situations. ${ }^{28-30}$ Technological innovations have enabled the monitoring of different tasks and activity levels more effectively and efficiently. ${ }^{30}$ Another benefit of sensor technologies is their ability to use large sample sizes with lower costs. ${ }^{29}$ However, adaptation of new technologies in healthcare settings requires positive attitudes toward technology, new skills among healthcare personnel and the appropriate support, especially for those who are less-motivated technology users ${ }^{31}$ or those who belong to older generations. ${ }^{32}$

To avoid redundancy and to ensure the value of the current review, ${ }^{33}$ we performed a comprehensive search for earlier systematic reviews of the Joanna Briggs Institute (JBI) Database of Systematic Reviews and Implementation Reports, PubMed and the Cochrane Database of Systematic Reviews, and found only five reviews that were related to personnel in any professional group. Khakurel $e t a l^{34}$ described a recent trend in wearable technology, and assessed both its potential in the work environment and challenges concerning the utilisation of wearables in the workplace. They identified a total of 359 articles, of which 34 met the selection criteria. The authors concluded that wearable technology can be used in the work environment for activities including monitoring, augmenting, assisting, delivering and tracking. Another review compared device-measured physical activity, sedentary behaviour and health across occupational groups, including healthcare workers. ${ }^{35}$ Two other reviews described physical activity in the workplace using both subjective and objective methods including research-grade accelerometers (eg, activPAL, Actigraph, GENEActiv), smartphone-integrated accelerometers, accelerometer-inclinometers and activity monitors (eg, Fitbit, Tractivity) ${ }^{36}{ }^{37}$ Further, Chappel $e t$ $a l^{38}$ assessed nurses' occupational physical activity levels using subjective and objective measures. The objective measurements used in the studies included heart rate monitoring, accelerometry, pedometry and direct observation.

We also screened ongoing reviews registered in the International Prospective Register of Systematic Reviews and found two ongoing systematic reviews on monitoring practices in workplace settings. Sands $e$ t $a l^{39}$ focus on best practices using wearable technologies to promote workplace physical activity, while Bustos et att aim to summarise progress in the development of physiological monitoring systems for occupational applications. However, we did not find any ongoing reviews focusing on different monitoring technologies used by healthcare personnel in the workplace. The gap in existing and ongoing review topics provides justification for a new review. $^{33}$

There are already numerous sensoring applications that offer potential benefits in healthcare settings. ${ }^{34}$ However, little is known about how these devices could be used to continually collect large-scale data to monitor healthcare personnel's health and well-being. Therefore, the overall aim of this scoping review is to examine sensor technology used to monitor the health, well-being and movement of healthcare personnel in healthcare settings. A scoping review is the best suited method for identifying certain characteristics of sensor technology and offering an overview of the nature and diversity of the knowledge available. ${ }^{41}{ }^{42}$ Introducing a categorised framework and the various purposes for which technological applications have been used could help us identify suitable applications for specific purposes and target groups, and thereby facilitate the adoption of devices in the workplace. ${ }^{34}$ The results of this scoping review will also highlight existing research gaps, ${ }^{43}$ allow for recommendations for future research and provide evidence on the best practices for sensor technology use in clinical settings. Further, this scoping review will be used as a preliminary step toward a systematic review and meta-analysis.

\section{METHODS AND ANALYSIS}

Aim

The overall aim of this scoping review is to examine what technologies are available to monitor the health, wellbeing and movement of healthcare personnel in healthcare settings. A scoping review of a body of literature is particularly useful for our topic, which has not yet been extensively reviewed. ${ }^{44}$ 


\section{Research questions}

In this review, we will address the following research questions:

1. What types of sensor technology have been used to measure health, well-being and movement among healthcare personnel in the workplace?

2. For what purposes has sensor technology been used to measure health, well-being and movement among healthcare personnel in the workplace?

3. How has sensor technology been used to measure health, well-being and movement among healthcare personnel in the workplace?

\section{Protocol registration}

A systematic review registration was submitted in Open Science Framework on 12 December 2020 (https://osf. io/smbxc/).

\section{Planned start and end date}

The review is planned to start on 1 December 2021 and end on 31 December 2022.

\section{Design}

In this study, a scoping review design will be used to form a conception of the use of monitoring technology among healthcare personnel in healthcare settings. As a foundation for the review, we will modify Arksey and O'Malley's ${ }^{45}$ framework for scoping reviews for our purposes. It fits into our review because it enables mapping the range of research data available for the topics that have not been previously reviewed and identifies gaps in the existing literature.

In this review, the updated framework by $\mathrm{JBI}^{42}$ with six stages will complement Arksey and O'Malley's ${ }^{45}$ approach: (1) identifying the research question; (2) identifying relevant studies; (3) selecting studies; (4) charting the data; (5) collating, summarising and reporting results; and (6) consulting with stakeholders. In reporting the scoping review, we will use the Preferred Reporting Items for Systematic Reviews and Meta-Analyses Extension for Scoping Reviews (PRISMA-ScR). ${ }^{46}$ The checklist of the PRISMA-ScR is attached as an online supplemental file 1. Guidance for conducting systematic scoping reviews has been used to complement our protocol. ${ }^{47}$

\section{Information sources and search strategy}

This scoping review will combine existing knowledge based on published empirical studies. A comprehensive literature search will be carried out using the following relevant bibliographical electronic databases: Web of Science (provides cross-disciplinary research on social science, science, technologies, humanities and the arts), PubMed, Medline, and PsycINFO in EBSCO, ScienceDirect (provides access to papers and articles on science and technology journals), Google Scholar, Cochrane, IET Electronic Library, the IEEE Xplore (a digital database of scholarly and technical literature, which provides the abstracts and complete texts of papers on computer science, electrical engineering and electronics), and
Elsevier/Engineering Village. These databases were selected as they cover scientific and technical literature and provide extensive insights into researchers' efforts in a wide range of relevant disciplines.

Search terms (or equivalent index terms and free-text words) for each database will be used to ensure a broad coverage of published studies in our review. A keyword search will be combined into a phrase search and include Boolean (AND, OR) terms. An example of the search terms to be used in PubMed is presented in table 1. A manual search will also be conducted with additional references by screening the reference lists of the included articles. In addition, specific journals related to the topic (eg, JMIR) will be searched manually. If a high number of studies are found using a hand search, the search strategy will be modified. ${ }^{48}$ Grey literature will not be used in this review.

\section{Citation management}

All citations will be imported into the web-based bibliographical manager EndNote V.X7 software to find and remove duplicates. Duplicate citations will be removed manually with further duplicates removed if found later in the process. Citations will be imported into the webbased systematic review software DistillerSR (Evidence Partners Incorporated, Ottawa, Ontario, Canada) for subsequent title and abstract relevance screening and data characterisation of full articles, if available.

\section{Eligibility criteria}

The review will be limited to texts, with an abstract available in English with no time restrictions. Peer-reviewed, published papers using a variety of designs and research methods will be included, as long as the paper includes a tested monitoring method. Papers describing the design process of the sensor technology, theoretical papers, statistical reviews, books or book chapters, letters, dissertations, editorials and study protocols will be excluded. More specifically, the review will be limited to certain studies as follows.

\section{Types of participants}

Any healthcare personnel, of any age and gender, with a variety of professional training, who are working in any healthcare area in patient care will be included in the review. Studies will be excluded if staff members did not have a role in patient care.

\section{Concept}

Type of sensor technology: in this review, sensor technology is understood as an objective measurement method for detecting quantifiable information. A sensor is a device that converts an input signal from a stimulus into a readable output signal. ${ }^{49}$ The input signal can be any measurable characteristic such as quantity or physical variation, while the output is ultimately an electrical signal. ${ }^{50}$ As sensors detect a variety of real-world properties and their proximity to a particular object, they can offer real-time monitoring, including detection and reporting. 


\begin{tabular}{|c|c|}
\hline$\#$ & \#Suchfrage \\
\hline$\# 1$ & "health personnel"(mh) OR "health personnel"[mh] \\
\hline \#2 & health care personnel[tiab] OR health personnel[tiab] \\
\hline \#3 & $\begin{array}{l}\text { (delivery of health care[tiab] OR (Health Care } \\
\text { Provider*[tiab]) OR (Healthcare Worker*[tiab]) }\end{array}$ \\
\hline \#4 & (\#1 OR \#2 OR \#3) \\
\hline \#5 & $\begin{array}{l}\text { "Electrical Equipment and Supplies"[mh] OR "Wearable } \\
\text { Electronic Devices"[mh] }\end{array}$ \\
\hline \#6 & "Monitoring, Physiologic"[mh] \\
\hline \#7 & "Monitoring, Ambulatory"[mh] \\
\hline \#8 & "Telemedicine ${ }^{\star ”[m h] ~}$ \\
\hline$\# 9$ & $\begin{array}{l}\text { (“Assistive Technology"[tiab]) OR (“Assistive } \\
\text { Technologies"[tiab]) OR ("Telecare"[tiab]) }\end{array}$ \\
\hline$\# 10$ & $\begin{array}{l}\text { ("Tele-health"[tiab]) OR (“Telemedicine"[tiab]) OR } \\
\text { ("telehomecare"[tiab]) OR (“tele-medicine"[tiab]) }\end{array}$ \\
\hline \#11 & (“ehealth"[tiab]) OR (“e-health"[tiab]) \\
\hline$\# 12$ & $\begin{array}{l}\text { ("vital signs monitoring"[tiab]) OR (“vital-signs } \\
\text { monitoring"[tiab]) OR ("vital signs"[tiab]) OR ("vital-signs or } \\
\text { monitoring"[tiab]) }\end{array}$ \\
\hline$\# 13$ & $\begin{array}{l}\text { (“mobile phone"(tiab)) OR ("cell phone"[tiab]) OR (“personal } \\
\text { digital assistant"[tiab]) OR ("personal smart assistant”[tiab]) }\end{array}$ \\
\hline \#14 & $\begin{array}{l}\text { ("Inertial sensor technology"[tiab] OR "motion sensor"” } \\
\text { [tiab] OR "movement sensor"”[tiab]) }\end{array}$ \\
\hline \#15 & ("Sensoring" [tiab] OR "Biosensing Techniques" [mh]) \\
\hline \#16 & $\begin{array}{l}\text { ((\#5 OR \#6 OR \#7 OR \#8 OR \#9 OR \#10 OR \#11 OR \#12 } \\
\text { OR \#13 OR \#14 OR \#15)) }\end{array}$ \\
\hline \#17 & "wellness"[tiab] OR "well-being"[tiab] \\
\hline \#18 & $\begin{array}{l}\text { "quality of life"[mh] OR "Health Status"[mh] OR "Personal } \\
\text { Satisfaction"[mh] }\end{array}$ \\
\hline \#19 & $\begin{array}{l}\text { "quality of life" [tiab] OR "Health Status" [tiab] OR } \\
\text { "Personal Satisfaction"[tiab] }\end{array}$ \\
\hline \#20 & (\#17 OR \#18 OR \#19) \\
\hline \#21 & Activit*[tiab] \\
\hline \#22 & $\begin{array}{l}\text { "physical activit"”[tiab] OR "activities of daily living"[tiab] or } \\
\text { "physical" activ"”[tiab] OR "free-living activity"[tiab] }\end{array}$ \\
\hline \#23 & (\#21 AND \#22) \\
\hline \#24 & Mobilit*[tiab] \\
\hline \#25 & "motor activit"”[tiab] OR "social mobilit"”[tiab] \\
\hline \#26 & "Social Mobility"[mh] \\
\hline \#27 & $\begin{array}{l}\text { "exp locomotion" [tiab] OR "running or walking"[tiab] } \\
\text { OR "ambulation or functional mobility"[tiab] OR } \\
\text { "movement"[tiab] }\end{array}$ \\
\hline \#28 & (\#24 OR \#25 OR \#26 OR \#27) \\
\hline \#29 & \#4 AND \#16 AND (\#20 OR \#23 OR \#28) \\
\hline
\end{tabular}

Monitored data can be detected by sensors and can then be sent for control and analysis. ${ }^{50}$

We will include any sensor technology used to measure health and well-being-related physiological outcomes at work, including vital signs, heart rate, blood pressure, temperature, respiratory rate, breathing rate and depth, energy expenditure, blood oxygenation or skin temperature as example. Physiological monitoring can include obesity and weight management or assessing sleep as long they are related to the work environment. Any studies involving invasive applications used to assess healthrelated physiological outcomes will be excluded. To describe movement, we will include sensor applications that measure, for example, motion, body motor activity, sedentary behaviour, body posture, step count, physical activity, geospatial activity, location variance or mobility in the workplace.

The type of sensor technology will be categorised as mechanical (eg, accelerometers, gyroscopes), optical (eg, fibre optic) or semiconductor sensors (temperature sensors) to assess different domains (healthcare, wellness or environmental domains) and networks (personal area, ambient/pervasive sensor networks, wide area networks). Sensor deployments may include physiological monitoring using telehealth or telemonitoring using bodyworn assessment applications (wearable or body-worn devices, physiological sensors, smart watches, etc). Other types of sensors can include ambient sensing, user touch point or consumer sensing applications. ${ }^{51}$

Sensor measurement units such as length (metre), mass (kilogram), time (seconds), electronic current (ampere), temperature (Celsius/Kelvin), energy (joule), acceleration $\left(\mathrm{m} / \mathrm{s}^{2}\right)$, area $\left(\mathrm{m}^{2}\right)$ and speed $(\mathrm{m} / \mathrm{s})$ will be identified. Characteristics of different types of sensor technology will be identified (active vs passive; digital vs analogue; null and deflection methods; input-output configuration).

Purpose of sensor technology: the purpose of sensor technology will be described (eg, 'to measure body temperature').

Use of sensor technology: a description of how sensor technology is used will be given (eg, pointing a thermometer toward the forehead and pressing the measurement button).

\section{Context}

We will include studies for which sensor technology has been used in any healthcare setting (primary healthcare, acute care, rehabilitation units, specialist services, etc) as long as the sensor system has been used during working hours. No limits for geographical, locational or cultural factors or racial, sex-based or discipline interests will be set. ${ }^{47}$ We will exclude any publications focusing only on free time (hobbies, running, nutrition monitoring, etc) not related to the work of healthcare professionals.

\section{Selection of sources of evidence}

The initial search will be performed by an information specialist to find articles related to the topic. Additional articles will be found using a manual search, for example, looking at the reference list of each article. After checking for duplicates, titles and abstracts will be first screened by two authors $(\mathrm{KH}, \mathrm{JC})$ for relevance to the topic and to see if they meet the inclusion criteria. In cases of discrepancy regarding inclusion or exclusion of a specific study, a third author (MV) will be consulted for a decision. Based on this process, 
potential full-text articles will be obtained. If access to any full-text article is lacking, we will contact the study's authors to obtain the full text or the findings of the study. A full-text review will be conducted to assess whether they meet inclusion criteria.

\section{Extracting and charting the results}

A coding manual aligned to the questions of the review will be developed to aid in the process of charting the data extracted from the papers. In addition, charting tables will be developed to show the results as a 'map' in a descriptive format that, again, will align to the questions of the review. The charting tables will be retested using three studies by all authors (MV, KH, JC, XH, JG, MSW) to ensure all relevant results are extracted. ${ }^{47}$ The review agreement will be evaluated using the overall kappa: ${ }^{52} \mathrm{a}$ kappa of greater than 0.8 will be considered to represent a high level of agreement. ${ }^{53}$

Relevant results from included papers will be extracted and inputted into predesigned charting tables by two authors $(\mathrm{KH}, \mathrm{JC})$. The process will be validated with the following steps and the guidance of MV. The authors (KH, JC) will first familiarise themselves with the study data. Second, they will independently extract data from the first three studies using the coding manual and pre-prepared tables. Third, the authors will meet and discuss their findings to determine whether their approaches to data extraction are consistent with each other's approaches, the research questions and the purpose of the review (led by MV). Fourth, the data extraction process will be approved as long as no uncertainties are found. ${ }^{54}$ If there are any discrepancies in the data extraction, the issue will be resolved appropriately depending on the specific question and expert area needed (MV, MSW).

All studies will be categorised using a two-step process. First, author, year, country, research setting, design, participant group and sample size will be extracted to describe the characteristics of the study. Second, to answer each research question, the following information will be extracted from the included study according to the research questions: (1) What technologies are used to monitor well-being, physical activity and movement among healthcare personnel?; (2) How are the technologies used? and (3) For what purposes are the technologies used?

Full-text articles to be included will be extracted by two authors. In cases of discrepancy, the issue will be resolved by a third author (MV). If there is any uncertainty related to the sensor technology used, it will be discussed with an expert in geoinformatics (MSW). Information and data collection relevant to answering the research questions will be determined by the reviewers collectively.

\section{Data synthesis}

Data synthesis includes collating, summarising and reporting the results. ${ }^{45}$ The data will be collated by combining numerical and thematic information in the data to summarise the background information of the studies. To answer the research questions, a thematic analysis in narrative format will be conducted. The reviewers will collectively produce an analysis process from the text data, and the themes will be formed according to the extracted tables. The data will be exported into SPSS V.27 (IBM Corp) for analysis. Descriptive statistics will be calculated to summarise the data, and frequencies and percentages will be used to describe nominal data. Any qualitative descriptions will be categorised using content analysis methods.

\section{Critical appraisal of individual sources of evidence}

The quality of each study will be appraised using designspecific appraisal tools. The Critical Appraisal Skills Programme checklists ${ }^{55}$ will be used for qualitative studies, the Strengthening the Reporting of Observational Studies in Epidemiology checklist for cohort, case-control and cross-sectional studies ${ }^{56}$ for quantitative studies, and the Cochrane Collaboration's tool ${ }^{57}$ for randomised trials. The quality of mixed-methods studies will be assessed using the Mixed Methods Appraisal Tool. ${ }^{58}$

\section{Patient and public involvement}

A stakeholder group including four nurse leaders working in healthcare settings has been formed for the scoping review. Their opinions will be consulted in different phases of the review process. First, the relevance of the topic of the review was confirmed before registration of the protocol. Second, the stakeholder group will participate in the study selection ${ }^{59-61}$ and supplement the data collected in the literature search by providing their experimental data ${ }^{61}$ or informing about any known unpublished studies. ${ }^{59}$ Third, these stakeholders will review emerging findings ${ }^{59}$ and provide input when interpreting the findings. ${ }^{59-61}$ Fourth, the stakeholders will provide preliminary feedback on the manuscripts, ${ }^{59} 61$ help identify key messages and relevant recommendations for practice and policymakers. Last, they will guide us in identifying the next steps toward future research. ${ }^{61}$ The opinions and feedback from the stakeholders will be collected through meetings, workshops, electronic surveys and focus group interviews. ${ }^{61}$

\section{ETHICS AND DISSEMINATION}

For this scoping review, research ethics approval will not be required. The findings from this scoping review will be disseminated through a peer-reviewed publication.

\section{DISCUSSION}

Although the health and well-being of healthcare personnel has received much research attention, there is a need for further assessment of these crucial factors associated with burden in health and well-being, specifically, research that is carried out in a timely, ongoing manner 
and with a large objective dataset. This study will generate evidence needed to explore what types of technology could be used in monitoring psychological and physical trajectories for health risk in the workplace. New sensor technologies could offer less intrusive and less burdening methods for assessing well-being and more objective methods for assessing physical activity.

Findings from randomised controlled trial (RCT) studies improving personnel health and well-being in the workplace have shown that trajectories affecting well-being at work are related to the life situations of the personnel, ${ }^{14}$ information that is difficult to capture retrospectively using survey forms only. ${ }^{12}{ }^{22}{ }^{26}$ However, measures to increase our understanding of the roles of specific events, emotional atmosphere and individuals' feelings or burden caused at work have not been given much attention. Muaremi $e t a l^{62}$ concluded that the use of wearable devices and smartphone applications can ensure better results than asking people about their moods in interviews or having them fill out questionnaires retrospectively. Therefore, one can argue that an ongoing data collection that records events and emotions in real time could be much more informative.

Due to the paucity of objective data, we intend to address any technologies used in any healthcare professional groups. We argue that the findings from this scoping review can play a vital role in selecting measures to support health promotion in the workplace. Our scoping review may also identify aspects of a future empirical study aiming to increase work efficiency, improve workers' physical well-being and reduce work-related injuries. ${ }^{34}$ Using objective measures is even more important in the current COVID-19 situation, when feasible and objective measures are needed to assess the well-being of healthcare personnel at their workplace.

As with any other scoping review, our study may have limitations. ${ }^{52}$ For example, there is the possibility that the review may have missed some relevant studies due to a selection of specific databases or due to the exclusion of grey literature from the search. The volume of articles identified may also be a key limiting factor. If not enough articles can be found, no conclusions can be made, and no recommendations can be offered to clinical practice or policymaking. On the other hand, if the search produces an exhaustive amount of studies, the balance between breadth and depth of analysis could be a challenge. However, since our review is not limited by time restraints, we aim to follow a rigourous review process. In addition, a critical appraisal of the included studies will be conducted in the study process and used to make reasonable recommendations for policy or practice. Further, a publication bias will be considered in the studies as follows. First, an over-representativeness of positive outcomes will be kept in mind in reporting the review results. ${ }^{63-65}$ Second, the funding body of each study (eg, industry funding) will be identified to declare any possible conflicts of interest. ${ }^{6465}$ Third, existing study registers (eg, ClinicalTrials.gov, osf. io, ISRCTN Registry) and protocols will be searched and screened to estimate the equivalence between the protocol and journal article. ${ }^{6465}$ We therefore hope that the findings from this scoping review can contribute to the body of knowledge on well-being and health among personnel, which will have a positive impact on clinical practice, research and policymaking in the area of health promotion in the workplace.

Acknowledgements We would like to thank Central South University, Xiangya School of Nursing and the Department of Nursing Science at the University of Turku for their support in the preparation of this protocol.

Contributors MV-conception (generator of the review) and responsible for the study design, searching preliminary literature for the background, initial search terms and writing the manuscript. $\mathrm{KH}$ - searching preliminary literature for the background and writing the manuscript. JC—commenting on the manuscript. $\mathrm{XH}-$ search strategy for papers and commenting on the manuscript. JG—commenting on the manuscript. MSW—-search strategy for papers related to monitoring devices and commenting on the manuscript.

Funding The work was supported by the Xiangya Nursing School, Central South University, China and the University of Turku, Department of Nursing Science, Finland. MSW would like to thanks the fundings supported by General Research Fund project id: 15603920, and Colloborative Research Fund project id: C402320GF, Hong Kong Research Grants Council.

Disclaimer The funding bodies do not have any role in the design, analysis, or interpretation or formation of conclusions of the results.

Competing interests None declared.

Patient and public involvement Patients and/or the public were involved in the design, or conduct, or reporting, or dissemination plans of this research. Refer to the Methods section for further details.

Patient consent for publication Not required.

Provenance and peer review Not commissioned; externally peer reviewed.

Supplemental material This content has been supplied by the author(s). It has not been vetted by BMJ Publishing Group Limited (BMJ) and may not have been peer-reviewed. Any opinions or recommendations discussed are solely those of the author(s) and are not endorsed by BMJ. BMJ disclaims all liability and responsibility arising from any reliance placed on the content. Where the content includes any translated material, BMJ does not warrant the accuracy and reliability of the translations (including but not limited to local regulations, clinical guidelines, terminology, drug names and drug dosages), and is not responsible for any error and/or omissions arising from translation and adaptation or otherwise.

Open access This is an open access article distributed in accordance with the Creative Commons Attribution Non Commercial (CC BY-NC 4.0) license, which permits others to distribute, remix, adapt, build upon this work non-commercially, and license their derivative works on different terms, provided the original work is properly cited, appropriate credit is given, any changes made indicated, and the use is non-commercial. See: http://creativecommons.org/licenses/by-nc/4.0/.

ORCID iDs

Maritta Välimäki http://orcid.org/0000-0001-7234-2454

Kirsi Hipp http://orcid.org/0000-0002-7007-9194

Jia Guo http://orcid.org/0000-0002-8304-9947

\section{REFERENCES}

1 WHO. Health workers. Available: https://www.who.int/occupational health/topics/hcworkers/en/ [Accessed 1 Apr 2020].

2 WHO. Global strategy on human resources for health: workforce 2030. Geneva World Health Organization; 2016. https://www.who.int/ hrh/resources/globstrathrh-2030/en/ [Accessed 1 Apr 2020].

3 OECD. Danish Ministry of health. health in the 21st century - high level policy Forum: Copenhagen 21 November 2019 issue notes. OECD, 2019. Available: https://www.oecd.org/health/health-21century/Policy-Forum-Issue-Notes.pdf [Accessed 1 Apr 2020].

4 Kieny M-P, Evans TG, Scarpetta S. Delivering quality health services: a global imperative for universal health coverage. Washington DC, US World Bank Group; 2018. http://documents.worldbank.org/ curated/en/482771530290792652/Delivering-quality-health-services- 
a-global-imperative-for-universal-health-coverage [Accessed $1 \mathrm{Apr}$ 2020].

5 Odendaal WA, Anstey Watkins J, Leon N, et al. Health workers perceptions and experiences of using mHealth technologies to deliver primary healthcare services: a qualitative evidence synthesis. Cochrane Database Syst Rev 2020;3:CD011942.

6 Figueroa CA, Harrison R, Chauhan A, et al. Priorities and challenges for health leadership and workforce management globally: a rapid review. BMC Health Serv Res 2019;19:239.

7 Aiken LH, Sermeus W, Van den Heede K, et al. Patient safety, satisfaction, and quality of hospital care: cross sectional surveys of nurses and patients in 12 countries in Europe and the United States. BMJ 2012;344:e1717.

8 West CP, Dyrbye LN, Erwin PJ, et al. Interventions to prevent and reduce physician burnout: a systematic review and meta-analysis. Lancet 2016;388:2272-81.

9 Wilkinson E. UK NHS staff: stressed, exhausted, burnt out. Lancet 2015;385:841-2.

10 Aiken LH, Sloane DM, Bruyneel L, et al. Nurse staffing and education and hospital mortality in nine European countries: a retrospective observational study. Lancet 2014;383:1824-30.

11 Dall'Ora C, Ball J, Reinius M, et al. Burnout in nursing: a theoretical review. Hum Resour Health 2020;18:41.

12 Hall LH, Johnson J, Watt I, et al. Healthcare staff wellbeing, burnout, and patient safety: a systematic review. PLOS One 2016;11:e0159015.

13 Ross-Walker C, Rogers-Clark C, Pearce S. A systematic review of registered nurses; experiences of the influence of workplace culture and climatic factors on nursing workloads. JBI Libr Syst Rev 2012;10:3080-145.

14 Trudel-Fitzgerald C, Chen Y, Singh A, et al. Psychiatric, psychological, and social determinants of health in the nurses' health study cohorts. Am J Public Health 2016;106:1644-9.

15 Joyce K, Pabayo R, Critchley JA, et al. Flexible working conditions and their effects on employee health and wellbeing. Cochrane Database Syst Rev 2010;2:CD008009.

16 Salmond SW, Begley R, Brennan J, et al. A comprehensive systematic review of evidence on determining the impact of magnet designation on nursing and patient outcomes: is the investment worth it? JBI Libr Syst Rev 2009;7:1119-78.

17 Cummings GG, MacGregor T, Davey M, et al. Leadership styles and outcome patterns for the nursing workforce and work environment: a systematic review. Int J Nurs Stud 2010;47:363-85.

18 Van der Heijden BIJM, Mulder RH, König C, et al. Toward a mediation model for nurses' well-being and psychological distress effects of quality of leadership and social support at work. Medicine 2017;96:e6505.

19 Kuehnl A, Seubert C, Rehfuess E, et al. Human resource management training of supervisors for improving health and wellbeing of employees. Cochrane Database Syst Rev 2019;9:CD010905.

20 Pekurinen V, Välimäki M, Virtanen M, et al. Work stress and satisfaction with leadership among nurses encountering patient aggression in psychiatric care: a cross-sectional survey study. Adm Policy Ment Health 2019;46:368-79.

21 Hopkins M, Fetherston CM, Morrison P. Aggression and violence in healthcare and its impact on nursing students: a narrative review of the literature. Nurse Educ Today 2018;62:158-63.

22 Kelly EL, Fenwick K, Brekke JS, et al. Well-Being and safety among inpatient psychiatric staff: the impact of conflict, assault, and stress reactivity. Adm Policy Ment Health 2016;43:703-16.

23 Salvagioni DAJ, Melanda FN, Mesas AE, et al. Physical, psychological and occupational consequences of job burnout: a systematic review of prospective studies. PLoS One 2017;12:e0185781.

24 Linton M-J, Dieppe P, Medina-Lara A. Review of 99 self-report measures for assessing well-being in adults: exploring dimensions of well-being and developments over time. BMJ Open 2016;6:e010641.

25 Scanlan JN, Still M. Relationships between burnout, turnover intention, job satisfaction, job demands and job resources for mental health personnel in an Australian mental health service. BMC Health Serv Res 2019;19:62.

26 Farquharson B, Bell C, Johnston D, et al. Nursing stress and patient care: real-time investigation of the effect of nursing tasks and demands on psychological stress, physiological stress, and job performance: study protocol. J Adv Nurs 2013;69:2327-35.

27 Islam MS, Baker C, Huxley P, et al. The nature, characteristics and associations of care home staff stress and wellbeing: a national survey. BMC Nurs 2017;16:22.

28 Ben-Zeev D, Scherer EA, Wang R, et al. Next-Generation psychiatric assessment: using smartphone sensors to monitor behavior and mental health. Psychiatr Rehabil J 2015;38:218-26.
29 Short CE, DeSmet A, Woods C, et al. Measuring engagement in eHealth and mHealth behavior change interventions: viewpoint of methodologies. J Med Internet Res 2018;20:e292.

30 L'Hommedieu M, L'Hommedieu J, Begay C, et al. Lessons learned: recommendations for implementing a longitudinal study using wearable and environmental sensors in a health care organization. JMIR Mhealth Uhealth 2019;7:e13305.

31 Anttila M, Välimäki M, Koivunen M, et al. Adoption of an Internetbased patient education programme in psychiatric hospitals. $J$ Psychiatr Ment Health Nurs 2011;18:914-23.

32 Ortega EJ, Recio Menéndez M, et al. Diffusion and usage patterns of Internet services in the European Union. Inf Res 2007;12.

33 Lund H, Juhl CB, Nørgaard B. Using an evidence-based research approach before a new study is conducted to ensure value. J Clin Epidemiol 2021:129:158-66.

34 Khakurel J, Melkas H, Porras J. Tapping into the wearable device revolution in the work environment: a systematic review. Inf Technol People 2018;31:791-818.

35 Prince SA, Elliott CG, Scott K, et al. Device-measured physical activity, sedentary behaviour and cardiometabolic health and fitness across occupational groups: a systematic review and meta-analysis. Int J Behav Nutr Phys Act 2019;16:30.

36 Buckingham SA, Williams AJ, Morrissey K, et al. Mobile health interventions to promote physical activity and reduce sedentary behaviour in the workplace: a systematic review. Digit Health 2019;5:205520761983988.

37 Shrestha N, Kukkonen-Harjula KT, Verbeek JH, et al. Workplace interventions for reducing sitting at work. Cochrane Database Syst Rev 2018;6:CD010912.

38 Chappel SE, Verswijveren SJJM, Aisbett B, et al. Nurses' occupational physical activity levels: a systematic review. Int J Nurs Stud 2017;73:52-62.

39 Sands C, Aguiar E, Tudor-Locke C. Best practices for using wearable technologies to promote workplace physical activity: a systematic review. PROSPERO; CRD42018102059, 2018. Available: https://www.crd.york.ac.uk/prospero/display_record.php?ID= CRD42018102059

40 Bustos LD, Guedes J, Torres Costa J. Evidencing the applicability of physiological monitoring for health management within occupational settings: protocol for a systematic review. Int J Occup Environ Health 2019;3:68-76.

41 Anderson S, Allen P, Peckham S, et al. Asking the right questions: Scoping studies in the commissioning of research on the organisation and delivery of health services. Health Res Policy Sys 2008;6:7.

42 Peters M, Godfrey C, Mclnerney P. Scoping reviews. In: Aromataris E, Munn Z, eds. JBI manual for evidence synthesis. The Joanna Briggs Institute, 2020.

43 Tricco AC, Lillie E, Zarin W, et al. A scoping review on the conduct and reporting of scoping reviews. BMC Med Res Methodol 2016;16:15

44 Mays N, Roberts E, Popay J. Synthesizing research evidence. In: Fulop N, Allen P, Clarke A, et al, eds. Studying the organisation and delivery of health services: research methods. London: Routledge, 2001: 188-219.

45 Arksey H, O'Malley L. Scoping studies: towards a methodological framework. Int J Soc Res Methodol 2005;8:19-32.

46 Tricco AC, Lillie E, Zarin W, et al. PRISMA extension for scoping reviews (PRISMA-ScR): checklist and explanation. Ann Intern Med 2018;169:467-73.

47 Peters MDJ, Godfrey CM, Khalil H, et al. Guidance for conducting systematic scoping reviews. Int J Evid Based Healthc 2015;13:141-6.

48 Vassar M, Johnson AL, Sharp A, et al. Citation bias in otolaryngology systematic reviews. J Med Libr Assoc 2021;109:62-7.

49 Gründler P. Chemical sensors: an introduction for scientists and engineers. Leipzig, Germany: Springer, 2007.

50 Askim JR, Mahmoudi M, Suslick KS. Optical sensor arrays for chemical sensing: the optoelectronic nose. Chem Soc Rev 2013;42:8649-82.

51 McGrath MJ, Ni Scanaill C, Nafus D. Sensor technologies: healthcare, wellness and environmental applications. Apress, 2013. https://www.apress.com/gp/book/9781430260134

52 Pham MT, Rajić A, Greig JD, et al. A scoping review of scoping reviews: advancing the approach and enhancing the consistency. Res Synth Methods 2014;5:371-85.

53 Dohoo IR, Martin W, Stryhn H. Methods in epidemiologic research. Charlottetown, Prince Edward Island: VER Inc, 2012.

54 Levac D, Colquhoun H, O'Brien KK. Scoping studies: advancing the methodology. Implementation Sci 2010;5:69. 
55 Critical Appraisal Skills Programme. CASP qualitative studies checklist, 2019. Available: https://casp-uk.net/casp-tools-checklists/ [Accessed 15 Aug 2021].

56 von Elm E, Altman DG, Egger M, et al. The strengthening the reporting of observational studies in epidemiology (STROBE) statement: guidelines for reporting observational studies. J Clin Epidemiol 2008;61:344-9.

57 Higgins JPT, Altman DG, Gøtzsche PC, et al. The Cochrane Collaboration's tool for assessing risk of bias in randomised trials. BMJ 2011;343:d5928.

58 Hong QN, Pluye P, bregues, S F. Mixed methods appraisal tool (MMAT) version 2018: user guide. Available: http://mixedmethods appraisaltoolpublic.pbworks.com/w/file/fetch/127916259/MMAT 2018_criteria-manual_2018-08-01_ENG.pdf [Accessed 15 Aug 2021].

59 Boote J, Baird W, Sutton A. Involving the public in systematic reviews: a narrative review of organizational approaches and eight case examples. J Comp Eff Res 2012;1:409-20.
60 Keown K, Van Eerd D, Irvin E. Stakeholder engagement opportunities in systematic reviews: knowledge transfer for policy and practice. $J$ Contin Educ Health Prof 2008;28:67-72.

61 Tricco AC, Zarin W, Rios P, et al. Engaging policy-makers, health system managers, and policy analysts in the knowledge synthesis process: a scoping review. Implementation Sci 2018;13:31.

62 Muaremi A, Arnrich B, Tröster G. Towards measuring stress with smartphones and wearable devices during workday and sleep. Bionanoscience 2013;3:172-83.

63 Dubben H-H, Beck-Bornholdt H-P. Systematic review of publication bias in studies on publication bias. BMJ 2005;331:433-4.

64 Joober R, Schmitz N, Annable L, et al. Publication bias: what are the challenges and can they be overcome? J Psychiatry Neurosci 2012;37:149-52.

65 Song F, Hooper L, Loke Y. Publication bias: what is it? How do we measure it? How do we avoid it? Open Access $J$ Clin Trials 2013;5:71-81. 